Long V. Cantines d'entreprise et discours hygiénistes dans l'industrie britannique de l'entre-deux-guerres. Le Mouvement Social 2014, 247(2), 65-83.

DOI link

https://doi.org/10.3917//ms.247.0065

ePrints link

http://eprint.ncl.ac.uk/247115

Date deposited

$16 / 04 / 2018$

Copyright

CC 2014 La Découverte 


\title{
CANTINES D'ENTREPRISE ET DISCOURS HYGIÉNISTES DANS L'INDUSTRIE BRITANNIQUE DE L'ENTRE-DEUX-GUERRES
}

\section{Vicky Long}

\author{
La Découverte | «Le Mouvement Social »
}

2014/2 n²47| pages 65 à 83

ISSN 0027-2671

ISBN 9782707181954

Article disponible en ligne à l'adresse :

https://www.cairn.info/revue-le-mouvement-social-2014-2-page-65.htm

\section{Pour citer cet article :}

Vicky Long, « Cantines d'entreprise et discours hygiénistes dans l'industrie britannique de l'entre-deux-guerres », Le Mouvement Social 2014/2 (n²47), p. 65-83.

DOI $10.3917 / \mathrm{lms} .247 .0065$

Distribution électronique Cairn.info pour La Découverte.

(C) La Découverte. Tous droits réservés pour tous pays.

La reproduction ou représentation de cet article, notamment par photocopie, n'est autorisée que dans les limites des conditions générales d'utilisation du site ou, le cas échéant, des conditions générales de la licence souscrite par votre établissement. Toute autre reproduction ou représentation, en tout ou partie, sous quelque forme et de quelque manière que ce soit, est interdite sauf accord préalable et écrit de l'éditeur, en dehors des cas prévus par la législation en vigueur en France. Il est précisé que son stockage dans une base de données est également interdit. 


\section{Cantines d'entreprise et discours hygiénistes dans l'industrie britannique de l'entre-deux-guerres}

par Vicky LoNG*

E Grande-Bretagne, l'implantation de cantines au sein des entreprises peut s'expliquer par les exigences des deux guerres mondiales ${ }^{1}$. Désireux de soutenir et d'accroître la production des secteurs industriels indispensables à l'effort de guerre, les pouvoirs publics ont en effet prescrit la création de cantines industrielles, conçues comme le moyen de préserver la santé des ouvriers, donc aussi leur rendement. C'est ainsi que le nombre de cantines a été multiplié par dix durant la Première Guerre mondiale ${ }^{2}$ et a connu de nouveau une forte progression entre 1939 et $1945^{3}$. Il est dès lors tentant d'interpréter l'instauration de cantines d'entreprise comme une composante de ce que John Pickstone appelle la "médecine productiviste", c'est-à-dire un système sanitaire, dominant dans la première moitié du XX $\mathrm{XX}^{\mathrm{e}}$ siècle, caractérisé par son souci « de la santé et de la vigueur de la main-d'œuvre et des forces armées ${ }^{4}$. On pourrait ainsi ne voir dans les cantines ouvertes pendant les deux guerres mondiales (souvent dans des usines de munitions) qu'un volet parmi d'autres du colossal investissement de l'État dans les infrastructures de l'industrie de l'armement, et ce à des fins militaires ${ }^{5}$.

Quelques cantines d'entreprise existaient avant 1914, à l'instar d'autres prestations sociales fournies par l'employeur. On peut avoir un aperçu de leur nature et de leur nombre à la lecture des rapports de l'inspection du travail ${ }^{6}$, corps institué par la loi de 1833 sur les manufactures et destiné à garantir que les employeurs se conforment à leurs obligations réglementaires. L'inspection se montre donc très intéressée par les politiques sociales spontanément mises en œuvre par les patrons. Ses rapports annuels préconisent la mise en place de cantines, dans lesquelles les

* Maître de conférences en histoire de la santé, Glasgow Caledonian University, The Centre for the Social History of Health and Healthcare. Traduction de Cécile Deniard.

1. Cet article est issu d'un projet d'étude financé par la fondation Wellcome ("The Politics and Practices of Health in Work, 1915-1951 ", bourse n076053/Z/04/Z), dirigé par le professeur Hilary Marland et le docteur Mathew Thomson et mené au Centre pour l'histoire de la médecine de l'université de Warwick.

2. Passant d'une centaine à environ un millier : voir Ministère DEs Munitions (ci-après MM), Health of Munition Workers Committee Final Report: Industrial Health and Efficiency, 1918, Command Papers (ci-après Cmd) 9065, p. 51-61.

3. Le nombre de cantines passa de 5695 en décembre 1941 à 8481 en décembre 1942 : Annual Report of the Chief Inspector of Factories for the Year 1943, 1944, Cmd 6563, p. 60.

4. J. Pickstone, "Production, Community and Consumption: the Political Economy of TwentiethCentury Medicine", in R. Cooter et J. Pickstone (eds.), Companion to Medicine in the Twentieth Century, Londres; New York, Routledge, 2003, p. 1-20, p. 2.

5. Voir D. Edgerton, Warfare State: Britain, 1920-1970, Cambridge, Cambridge University Press, 2006, p. 77-83.

6. Pour une histoire de l'inspection du travail, voir E. CROoKs, The Factory Inspectors: a Legacy of the Industrial Revolution, Stroud, Tempus, 2005. 
ouvriers auraient la possibilité d'acheter et de consommer de la nourriture, ainsi que de salles à manger, c'est-à-dire de locaux, au sein de l'entreprise, où ils pourraient manger le repas apporté de chez eux. En l'absence de telles dispositions, les inspecteurs craignent que les ouvriers ne mettent leur santé en péril en se contentant de rations alimentaires insuffisantes, réchauffées dans les fourneaux employés aux opérations de fabrication et avalées à la va-vite au milieu des rebuts de la production industrielle. Ces conditions étaient jugées particulièrement préoccupantes dans les industries utilisant des matières premières nocives : un inspecteur déplore, en 1892, que dans la salle à manger d'une usine d'arsenic londonienne, " fort sale ", on trouve une "bonne quantité de déchets " ${ }^{7}$. En 1893, la commission de la céruse préconise de rendre obligatoire l'existence d'une salle à manger séparée ${ }^{8}$. Ces constats et recommandations traduisent une volonté étatique croissante d'encadrer les procédés industriels utilisant des matières premières à l'origine de maladies professionnelles spécifiques de plus en plus reconnues?.

Dans ces industries insalubres mais aussi ailleurs, des patrons jouissant d'une réputation de philanthropes mettaient également des cantines et des salles à manger à disposition de leur personnel. Dans la cité ouvrière de Port Sunlight, planifiée par Lever Brothers, où se déploie un important programme d'œuvres sociales, on trouve par exemple des restaurants séparés pour la main-d'œuvre féminine et masculine $\mathrm{e}^{10}$, dans la lignée de certains patrons paternalistes de la première moitié du XIX siècle comme Robert Owen, chez qui les considérations humanitaires s'associaient au désir d'accroître le rendement ${ }^{11}$. Si le paternalisme passe de mode dans les premières années du $\mathrm{XX}^{\mathrm{e}}$ siècle, les valeurs chrétiennes en vigueur dans certaines entreprises continuent à inspirer la mise à disposition plus systématique d'équipements de confort ${ }^{12}$. L'inspecteur du Warwickshire observe d'ailleurs que les installations offertes au personnel, notamment les salles à manger, suscitent " un lien de sympathie et de bienveillance entre le patron et ses employés » ${ }^{13}$. En 1913, le rapport annuel de l'Inspection du travail note que les employeurs ont de plus en plus " conscience que la condition physique de l'ouvrier a un retentissement important sur la production de la manufacture ", d'où "une lente généralisation des salles à manger et restaurants ". Le texte cite l'exemple d'une entreprise de Bristol où il a été constaté que les ouvriers tombaient deux fois moins malades depuis l'ouverture de restaurants dans ses usines ${ }^{14}$. Ces cas exemplaires sont cependant loin d'être légion.

7. MM, Report of the Chief Inspector of Factories and Workshops to Her Majesty's Principal Secretary of State for the Home Department, for the year ending $31^{\text {st }}$ October 1892, 1893, Cmd 6978, p. 22.

8. MM, Report of the Chief Inspector of Factories and Workshops to Her Majesty's Principal Secretary of State for the Home Department, for the year 1893, 1894, Cmd 7368, p. 50.

9. Pour une vue d'ensemble, voir P. W. J. Bartrip, The Home Office and the Dangerous Trades: Regulating Occupational Disease in Victorian and Edwardian Britain, Amsterdam ; New York, Rodopi, 2002.

10. W. L. George, Labour and Housing at Port Sunlight, Londres, A. Rivers, 1909, p. 46-51.

11. I. Donnachie, Robert Owen: Owen of New Lanark and New Harmony, East Linton, Tuckwell Press, 2000, p. 127.

12. Voir R. Fitzgerald, "Employment Relations and Industrial Welfare in Britain: Business Ethics versus Labour Markets", Business and Economic History, Vol. 28, 1999, p. 167-179.

13. MM, Report of the Chief Inspector of Factories and Workshops to Her Majesty's Principal Secretary of State for the Home Department, for the year ending 31 $1^{\text {st }}$ October 1890, 1891, Cmd 6330, p. 22.

14. MM, Annual Report of the Chief Inspector of Factories and Workshops for the Year 1913, 1914, Cmd 7491, p. 8. 
Selon le même rapport, les établissements « où des denrées parfaitement cuisinées et s'inscrivant dans un régime nutritionnel bien pensé et généreux seront servies à prix coûtant dans un réfectoire agréable resteront sans doute toujours l'exception ». Considérant la généralisation des cantines d'entreprise comme un idéal inaccessible, les inspectrices suggèrent qu'il serait plus efficace d'inciter les employeurs à mettre à la disposition des ouvriers des salles à manger où ils pourraient consommer la nourriture qu'ils auraient apportée, d'autant que « dans la grande majorité des usines et ateliers, on ne trouve toujours aucun local réellement satisfaisant où prendre un repas correct pendant les pauses $»^{15}$. Même si certains patrons de la fin du XIX et du début du XX $\mathrm{X}^{\mathrm{e}}$ siècle mettent en œuvre une politique d'action sociale (logements ouvriers, pensions de retraite, indemnités de maladie), les mesures prises ne sont guère efficaces pour diminuer la prévalence des maladies d'origine professionnelle. Leur objectif est surtout d'accroître le rendement ou de restaurer la domination du chef d'entreprise dans certains secteurs industriels particuliers ${ }^{16}$. En dehors de ces secteurs, la plupart ne peuvent se convaincre que les efforts financiers consentis à cette fin ne sont pas superflus et qu'ils seront compensés par une augmentation du rendement de la main-d'œuvre et de la production ${ }^{17}$.

Au vu de la quasi-inexistence des cantines d'entreprise avant 1914 et de leur rapide multiplication pendant le conflit, la littérature sur le sujet s'est jusqu’à présent concentrée sur les fonctions que celles-ci ont pu remplir dans la société britannique en guerre, laissant entendre qu'elles n'ont plus joué qu'un rôle négligeable une fois la paix revenue. James Vernon, par exemple, a récemment affirmé qu'au lendemain de l'armistice, " le rêve utopique de voir une cantine au cœur de chaque usine $[\ldots]$ se dissipa rapidement ${ }^{18}$, les cantines n'étant pas la priorité des entreprises confrontées à la dépression des années 1920 et 1930 . Pourtant, elles ne disparaissent pas pendant l'entre-deux-guerres, au contraire. Pour expliquer cette persistance - et même cette croissance - durant ces vingt années, le rôle qu'elles ont joué dans les rapports sociaux est donc à réinterroger.

Après avoir mis en lumière les objectifs des institutions gouvernementales qui encouragèrent la création de cantines d'entreprise durant les deux guerres mondiales, cet article avancera donc deux arguments pour expliquer que certains patrons aient, de leur propre chef et à leurs frais, continué à faire fonctionner des cantines. On montrera d'abord que les cantines ont les faveurs des industriels parce qu'elles incarnent une conception englobante de la santé au travail et permettent aux patrons de se dédouaner de leur responsabilité dans la maladie de leurs ouvriers et de repousser l'adoption d'une législation sur l'hygiène et la sécurité au travail. Cette attitude n'est pas sans rappeler celle des hygiénistes français du XIXe siècle pour qui la mécanisation industrielle était porteuse d'une amélioration de la santé des ouvriers,

15. Ibid., p. 81. Sur le travail des inspectrices, voir H. Jones, "Women Health Workers: The Case of the First Women Factory Inspectors", Social History of Medicine, Vol. 1, No. 2, 1988, p. 165-181.

16. R. Fitzgerald, British Labour Management and Industrial Welfare, 1846-1939, Londres; New York, Croom Held, 1988, p. 58-67 et 84-89.

17. Voir A. J. McIvor, "Employers, the Government, and Industrial Fatigue in Britain, 18901918”, British Journal of Industrial Medicine, Vol. 44, No. 11, 1987, p. 724-732, citation p. 729-730.

18. J. Vernon, Hunger: a Modern History, Cambridge (Massachusetts) ; Londres, The Belknap Press of Harvard University, 2007, p. 165-166. 
essentiellement menacée par leur mode de vie hors des murs de l'atelier ${ }^{19}$, comme le prétend en 1840 Louis René Villermé ${ }^{20}$. En Grande-Bretagne, cette approche englobante de l'hygiène industrielle connaît un regain pendant la Première Guerre mondiale et l'entre-deux-guerres, ce qui constitue un recul par rapport aux mesures prises par l'État à la fin du XIX ${ }^{\mathrm{e}}$ siècle $^{21}$.

Cet article défend également l'idée qu'on peut lire dans l'évolution des formes et des fonctions des cantines industrielles de l'entre-deux-guerres une réorientation des objectifs de la politique sociale d'entreprise, à l'heure où les valeurs du paternalisme philanthropique sont progressivement abandonnées au profit de méthodes de gestion scientifiques. Ainsi, dans les deux premières décennies du $\mathrm{XX}^{\mathrm{e}}$ siècle, les promoteurs d'une politique sociale de l'entreprise présentaient la cantine comme un antidote à l'atmosphère impersonnelle de l'usine. À partir des années 1930, les cantines deviennent au contraire l'incarnation même de la volonté de rationalisation industrielle, elles sont censées à la fois brider les comportements indisciplinés et assurer l'entretien matériel du facteur humain de la production industrielle.

\section{La cantine d'entreprise pendant la guerre}

La Première Guerre mondiale voit la multiplication rapide du nombre de cantines d'entreprise. Une centaine avant le conflit, elles sont un millier lorsqu'il s'achève et nourrissent alors environ un million d'ouvriers ${ }^{22}$. Fruit d'une volonté gouvernementale, cette croissance s'intègre dans un train de mesures plus global visant à répondre aux nécessités de la guerre. Celle-ci, qui place la production au premier rang des priorités, modifie en effet la composition de la population active. À mesure que les hommes partent pour le front, ils sont remplacés par des adolescents et des femmes. Une partie de la nouvelle main-d'œuvre ouvrière est issue de la domesticité ou d'autres formes de travail en usine ${ }^{23}$, mais une proportion importante n'appartenait pas à la population active au début du conflit : les femmes représentaient ainsi $26,5 \%$ de la population active en $1915-1916$, et 46,9 \% en 1916-1917 ${ }^{24}$.

La guerre entraînant une augmentation considérable du temps de travail dans de nombreuses usines, le gouvernement met sur pied plusieurs organismes chargés d'une réflexion sur les moyens d'en limiter les effets néfastes sur la santé des ouvriers. En 1915, le ministère des Munitions constitue une commission sanitaire pour formuler des propositions "sur les questions d'usure professionnelle, de temps de

19. Voir T. Le Roux, "Hygienists, Workers' Bodies and Machines in Nineteenth-Century France", Revue européenne d'histoire, Vol. 20, No. 2, 2013, p. 255-270.

20. L. R. Villermé, Tableau de l'état physique et moral des ouvriers [1840], commenté dans W. Coleman, Death is a Social Disease: Public Health and Political Economy in Early Industrial France, Madison, The University of Madison Press, 1982, p. 205-238, p. 220.

21. Voir A. S. Wohl, Endangered Lives: Public Health in Victorian Britain, Londres, J. M. Dent \& Sons, 1983 , p. $257-284$.

22. MM, Health of Munition Workers Committee Final Report..., op. cit., p. 51-61. L'évaluation du nombre d'employés ayant accès à une cantine est tirée de J. M. WInTER, The Great War and the British People [1985], Basingstoke, Palgrave Macmillan, 2003, p. 211.

23. G. Braybon, Women Workers in the First World War, Londres, Routledge, 1981, p. 48-50.

24. D. Tном, Nice Girls and Rude Girls: Women Workers in World War I, Londres, I. B. Tauris, 2000, p. 34 . 
travail et autres sujets affectant la santé individuelle et le rendement des ouvriers ${ }^{25}$ dans les usines de munitions. Après s'être rendue dans un certain nombre d'établissements, elle rédige vingt et une notes et deux rapports. En 1916, le même ministère se dote d'un département d'action sociale dont la direction est confiée au sociologue Benjamin Seebohm Rowntree (1871-1954), avec pour mission de mettre en œuvre les recommandations de la commission sanitaire.

Si la législation industrielle d'avant 1914 avait pour principal objet de limiter le temps de travail des femmes et des enfants et d'interdire leur emploi dans des métiers exposés à un risque élevé de maladie professionnelle ${ }^{26}$, la guerre voit naître un intérêt sans précédent pour la promotion de la santé des travailleurs. Cet interventionnisme d'un genre nouveau se caractérise par son insistance sur les besoins physiologiques des ouvriers, en particulier sur leurs besoins nutritionnels, au détriment des risques proprement professionnels. Dans cet esprit la commission sanitaire du ministère des Munitions affirme que " ce sont les conditions de travail plutôt que la nature même des travaux qui sapent la force physique et l'endurance de l'ouvrier " ${ }^{27}$. En GrandeBretagne comme ailleurs, cet argumentaire n'est pas entièrement nouveau ${ }^{28}$, mais l'attitude de la commission témoigne ainsi de la montée en puissance de la médecine préventive, mettant au cour de son discours la santé plutôt que la maladie et insistant sur la nécessité d'étudier les individus au sein de leur environnement. Sir George Newman, président de cette commission sanitaire, partisan de la création d'un service médical d'État, affirme dans son rapport final :

«La science et l'art de la Médecine ne se limitent pas à diagnostiquer et traiter la maladie. [...] C'est bien plutôt la science et l'art de la Santé, de la façon dont l'homme peut apprendre à mener une existence saine, au summum de ses capacités physiques et intellectuelles, [...] jouissant de sa pleine puissance de travail. [...] L'individu [...] ne résume d'ailleurs pas à lui seul le problème. Il faut considérer son histoire personnelle, son hérédité, sa famille, sa vie domestique, ses mœurs, son logement autant que son lieu de travail [...]. La productivité industrielle, dans ses rapports avec la santé et l'usure au travail, est donc dans une large mesure un sujet de médecine préventive, une question de physiologie et de psychologie, de sociologie et de gestion de la main-d'œuvre $»^{29}$.

25. MM, Health of Munition Workers Committee Interim Report: Industrial Efficiency and Fatigue, 1917, Cmd 8511, p. 2.

26. Voir P. W. J. Bartrip, The Home Office and the Dangerous Trades..., op. cit. ; B. Harrison, Not only the 'Dangerous Trades': Women's Work and Health in Britain, 1880-1914, Londres, Taylor \& Francis, 1996 ; C. Malone, Women's Bodies and the Dangerous Trades in England, 1880-1914, Woodbridge, Boydell \& Brewer, 2003.

27. MM, Health of Munition Workers Committee Final Report..., op. cit., p. 11.

28. Par exemple, en 1902, Thomas Oliver soulignait les risques sanitaires liés au surmenage en général dans un article pourtant inclus dans un recueil consacré aux risques sanitaires induits par les procédés et matériaux employés dans certaines industries. Voir T. Oliver, "The Physiology and Pathology of Work and Fatigue", in T. Olrver (ed.), Dangerous Trades: The Historical, Social, and Legal Aspects of Industrial Occupations as Affecting Health, by a Number of Experts, Londres, John Murray, 1902, p. 104-117. Ces arguments rejoignaient aussi, dans une certaine mesure, les opinions défendues par les hygiénistes français du XIX ${ }^{e}$ siècle. Voir W. Coleman, Death is a Social Disease..., op. cit., p. 205-238, et T. Le Roux, "Hygienists, Workers' Bodies and Machines...", art. cité.

29. MM, Health of Munition Workers Committee Final Report..., op. cit., p. 15. 
Il reprend ce passage dans une note de 1919 à l'intention du ministère de la Santé récemment créé, ce qui démontre à quel point cette nouvelle approche englobante des questions sanitaires a imprégné la politique gouvernementale pendant la guerre $^{30}$. Son influence s'est étendue à d'autres pays, comme le montrent les travaux, à cette époque, de Joseph Schereschewsky, employé au Service de santé publique des États-Unis, qui minimisent l'influence des facteurs professionnels sur la santé des salariés pour se concentrer sur l'impact de maladies bénignes et infectieuses contractées sur le lieu de travail $^{31}$.

En raison de l'influence grandissante des milieux scientifiques sur les politiques gouvernementales après le début du conflit ${ }^{32}$, la commission sanitaire du ministère des Munitions se détourne d'une vision paternaliste de l'action sociale pour adopter une approche qui met davantage en évidence l'impact de l'environnement au travail et du temps de travail sur l'état de santé physique des ouvriers, ce qui, en dernière analyse, affecte la productivité. Les mesures prises pendant la guerre reflètent donc l'attention croissante portée à la dimension incarnée du travail industriel ${ }^{33}$. La machine doit être au service de l'homme, conformément à ce que la commission sanitaire appelle le "fondement physiologique du travail ${ }^{34}$. En même temps et paradoxalement, le corps de l'ouvrier est souvent comparé à une machine qui a besoin d'un "carburant " approprié. Le comité d'alimentation explique ainsi en 1917 que «le rendement du travailleur manuel dépend aussi directement de son alimentation que le rendement d'un moteur de son essence $»^{35}$.

L'importance que la commission sanitaire accorde à la diététique au sein des cantines d'entreprise témoigne aussi des progrès accomplis par les sciences de la nutrition. Après s'être focalisées sur l'apport énergétique et protéinique des aliments, celles-ci commencent à prendre en compte le rôle des vitamines dans la santé ${ }^{36}$. Les cantines soutiennent la productivité en prévenant l'anémie, la malnutrition et l'épuisement au travail, mais tiennent également lieu de substitut à la cuisine familiale, à une époque où de nombreux salariés travaillent trop loin de chez eux pour rentrer déjeuner. Le gouvernement regarde donc les cantines comme la mesure-phare des programmes d'action sanitaire et sociale. Dans un rapport établi pour la commission sanitaire en 1917, le docteur T. H. Agnew souligne que «la qualité, la quantité et le rythme de la production dépendent dans une large mesure de l'alimentation

30. Sir G. Newman, An Outline of the Practice of Preventive Medicine: A Memorandum addressed to the Minister of Health, 1919, Cmd 363, p. 6-7. Cette note est commentée dans S. STURDy, "Hippocrates and State Medicine: George Newman outlines the Founding Policy of the Ministry of Health", in C. Lawrence et G. WeIsz (eds.), Greater than the Parts: Holism in Biomedicine, 1920-1950, New York ; Oxford, Oxford University Press, 1998, p. 112-134.

31. C. S. Sellers, Hazards of the Job: From Industrial Disease to Environmental Health Science, Chapel Hill ; Londres, University of North Carolina Press, 1997, p. 107-140.

32. D. F. Sмітн, "Nutrition Science and the two World Wars", in D. F. Sмiтн (ed.), Nutrition in Britain: Science, Scientists and Politics in the Twentieth Century, Londres, Psychology Press, 1997, p. $142-165$.

33. C. Wolkowitz, Bodies at Work, Londres, Sage, 2006, p. 54-69.

34. MM, Health of Munition Workers Committee Final Report..., op. cit., p. 7.

35. Comité d'alimentation, réunion du 24 avril 1917, cité dans D. F. SMITH, "Nutrition Science...", art. cité, p. 148. Voir également A. Rabinbach, Le moteur humain: l'énergie, la fatigue et les origines de la modernité, trad. de l'anglais par M. Luxembourg, Paris, La Fabrique, 2004.

36. D. F. Sмітн, "Nutrition Science...", art. cité, p. 143. 
des ouvriers ${ }^{37}$, message repris dans un manuel de 1921 qui insiste sur les bénéfices physiologiques des cantines, susceptibles de prévenir les maladies et les arrêts de travail. Il prévient aussi que " faute de fournir le minimum d'énergie nécessaire sous la forme d'une nourriture appétissante et digeste, toutes les autres mesures d'hygiène appliquées à la vie des ouvriers n'auront aucune portée $»^{38}$.

Les cantines d'usine sont également considérées comme un moyen de pallier les insuffisances de l'alimentation des ouvriers en dehors de l'entreprise. Même si la pénurie de vivres frappe moins sévèrement la Grande-Bretagne que d'autres pays sur le continent, une certaine « hiérarchie alimentaire " prévaut pendant la guerre ${ }^{39}$. En encourageant la création de cantines sur le lieu de travail, le gouvernement se dote donc d'un outil supplémentaire pour intervenir dans la répartition et la gestion des ressources alimentaires ${ }^{40}$. Un parallèle peut être établi entre les mesures visant à fournir un ravitaillement adéquat aux soldats du front ${ }^{41}$ et celles qui cherchent à nourrir les ouvriers chargés de leur procurer des munitions. L'usage fréquent de métaphores militaires appliquées aux ouvriers de l'industrie témoigne d'ailleurs des analogies perçues entre les deux groupes. "Si l'on "ne se bat pas le ventre vide", n'est-il pas vrai également que l'on ne travaille pas le ventre vide ?", demande en 1914 l'inspectrice du travail Rose Squire ${ }^{42}$.

Pendant la guerre, les préoccupations utilitaires semblent l'emporter dans la construction des cantines. Un rapport du ministère des Munitions recommande d'en ouvrir dans des bâtiments temporaires afin de répondre aux exigences du moment. De façon très pratique, il évoque l'usage de sols en béton, l'aménagement d'allées entre les tables de manière à ce que les ouvriers puissent aller et venir commodément, l'installation de barrières devant le comptoir de service pour prévenir les bousculades ${ }^{43}$. En complément ou parfois en remplacement des cantines, certaines usines font circuler des chariots pour distribuer boissons et collations ou bien proposent des buvettes. Les efforts nécessaires pour surmonter le défi logistique que représente la fourniture de repas à un grand nombre d'ouvriers sur le lieu de production peuvent, jusqu’à un certain point, être rapprochés de la difficulté de nourrir les soldats du front, où les problèmes liés à l'approvisionnement, à la préparation des aliments et à leur transport conduisent à proposer des repas monotones et rebutants

37. MM, Health of Munition Workers Committee Interim Report..., op. cit., p. 104.

38. E. L. Collis et M. Greenwood, The Health of the Industrial Worker, Londres, J. \& A. Churchill, 1921, p. 252. Médecin, Collis avait été inspecteur du travail et membre de la commission sanitaire du ministère des Munitions, tandis que Greenwood appartenait au comité de recherche sur l'usure au travail créé après la dissolution de la commission sanitaire.

39. Ina Zweiniger-Bargielowska parle de "hiérarchie alimentaire " pour contester l'idée que les vivres étaient équitablement répartis pendant la Seconde Guerre mondiale en Grande-Bretagne. Voir I. Zweiniger-Bargielowska, "Fair Shares? The Limits of Food Policy in Britain during the Second World War", in I. Zweiniger-Bargielowska, R. Duffett et A. Drouard (eds.), Food and War in Twentieth-Century Europe, Farnham, Burlington, 2011, p. 125-138. Voir également ID., Austerity in Britain: Rationing, Controls, and Consumption, 1939-1955, Oxford, Oxford University Press, 2000.

40. I. Zweiniger-Bargielowska, R. Duffett et A. Drouard (eds.), Food and War..., op. cit.

41. Voir R. Duffett, "British Army Provisioning on the Western Front, 1914-1918", in I. Zweiniger-Bargielowska, R. Duffett et A. Drouard (eds.), Food and War..., op. cit., p. 27-39.

42. MM, The Annual Report of the Chief Inspector of Factories and Workshops for the Year 1914, Cmd 7491, p. 52.

43. MM, Canteen Construction and Equipment, Cmd 8199, 1916. Commenté dans V. Long, "Industrial Homes, Domestic Factories: the Convergence of Public and Private space in Interwar Britain”, Journal of British Studies, Vol. 50, No. 2, 2011, p. 443-464, p. 444-445. 
qui, s'ils procurent une ration calorique suffisante, ne constituent en aucun cas un idéal du point de vue de la santéf ${ }^{44}$. En pratique, le corned-beef et les biscuits sont les principaux éléments des rations militaires, du fait de leur durée de conservation, de leur apport énergétique et de leur facilité de transport. Le manque de légumes provoque des carences en vitamine $\mathrm{C}$, tandis que la pénurie d'eau fraîche pour faire descendre ces aliments secs et bourratifs oblige les soldats à boire l'eau des flaques, au risque d'entretenir le cycle de la dysenterie et de la gastroentérite ${ }^{45}$.

La croissance rapide du nombre de cantines industrielles pendant la guerre peut apparaître comme une mesure pragmatique visant à préserver la santé physique et la productivité des ouvriers. On peut aussi y déceler une connotation disciplinaire, une forme de restauration collective apparentée aux cantines scolaires et aux cantines populaires, lesquelles s'appuyaient elles-mêmes sur des pratiques mises au point dans des environnements disciplinaires comme les prisons, les asiles et les hospices ${ }^{46}$. Si la connotation punitive s'estompe, la vocation disciplinaire perdure. Cette interprétation est d'autant plus valable qu'une grande partie du mouvement en faveur de la création de cantines pendant la Première Guerre mondiale résulte d'initiatives de la commission "cantine " du Bureau central de contrôle ${ }^{47}$, qui les considère comme une manière constructive de réfréner la consommation d'alcool ${ }^{48}$. Plusieurs auteurs soulignent ainsi l'effet salutaire que les cantines peuvent avoir sur le comportement des ouvriers. Les rédacteurs d'un texte publié peu après la guerre avertissent le lecteur que des tables sans apprêts et une vaisselle rudimentaire "ne feront qu'encourager le manque de soin, la casse et les mauvaises manières "; à l'inverse, une cantine agrémentée de rideaux, de tableaux, de couverts, d'une vaisselle en porcelaine, de nappes propres, de fleurs et, idéalement, d'une vue au sud sur un terrain cultivé, rehaussera "tout à fait la tenue du personnel de l'usine ${ }^{49}$. Un certain nombre de cantines sont conçues avec une vue sur l'extérieur, permanence d'une idée qui sous-tendait déjà la conception des parcs urbains de l'époque victorienne : le spectacle d'un paysage domestiqué doit contribuer au bien-être physique et moral des classes ouvrières urbaines ${ }^{50}$.

\section{Cantines et hygiène industrielle dans l'entre-deux-guerres}

Si la convergence des besoins militaires et industriels explique la multiplication des cantines pendant la guerre, on pourrait s'attendre à une diminution de leur nombre avec le retour à la paix, d'autant que l'heure est à un ralentissement économique qui voit le taux de chômage augmenter et les entreprises peiner à reprendre leur activité.

En l'absence de statistiques officielles, il est difficile d'évaluer avec certitude le nombre des cantines d'entreprises pendant l'entre-deux-guerres. L'effectif de

44. R. Duffett, "British Army Provisioning...", art. cité.

45. Ibid., p. 33-37.

46. J. Vernon, Hunger: a Modern History, op. cit.

47. Le Food (War) Committee a été mis en place par la Royal Society en 1915 pour conseiller les organismes gouvernementaux chargés de réguler la politique alimentaire, la distribution et le rationnement.

48. J. M. Winter, The Great War..., op. cit., p. 210-211.

49. E. L. Collis et M. Greenwood, The Health of the Industrial Worker..., op. cit., p. 261.

50. Voir C. Hickman, "'To brighten the aspect of our streets and increase the health and enjoyment of our city': The National Health Society and urban green space in late-nineteenth century London", Landscape and Urban Planning, Vol. 118, October 2013, p. 112-119. 
l'inspection du travail passe de 138 inspecteurs en 1901 à 290 en 1937. Ils ont pour mission de contrôler les conditions de travail dans 266811 entreprises dont 240383 usines et ateliers. On ne s'étonnera donc pas des difficultés qu'ils ont rencontrées pour faire appliquer la réglementation et, plus encore, rendre compte du progrès des prestations sociales spontanément accordées par les employeurs ${ }^{51}$. Néanmoins, l'examen des activités des entreprises considérées montre qu'un certain nombre de patrons continuent à investir dans les cantines après la fin des hostilités, et que, pour reprendre la formule de James Vernon, le "rêve utopique " ${ }^{52}$ d'une cantine dans chaque usine ne s'est pas complètement dissipé. Ainsi, l'entreprise de construction navale Beardmore, qui avait mis des salles à manger à la disposition de ses employés dès les années 1900 et considérablement investi dans des équipements sociaux pendant la guerre, continue sur cette lancée pendant les années 1918-1919. Joseph Melling a pu en conclure que l'entreprise faisait le choix de garder des équipements sociaux luxueux afin de retenir sa main-d'œuvre ${ }^{53}$. Les rapports annuels de l'inspection ne manquent d'ailleurs pratiquement jamais de souligner l'augmentation du nombre de cantines. Celui de 1934 indique que, dans certaines régions, « les ouvriers ont tellement l'habitude de trouver ce type de service qu'ils pensent que son absence est nécessairement illégale ${ }^{54}$. Fin 1941, les chiffres collectés montrent que leur nombre s'élève à 5695 contre un millier en $1918^{55}$. Certes, ce chiffre comprend plusieurs cantines créées après le déclenchement de la guerre en 1939, mais il confirme qu'au cours de l'entre-deux-guerres, nombre d'employeurs ont décidé de conserver les cantines ouvertes pendant le conflit précédent tandis que d'autres en ont aménagé de nouvelles.

Ce n'est d'ailleurs pas la seule mesure sociale que plusieurs employeurs pérennisent quoiqu'elle ait perdu après 1918 son caractère obligatoire. Beaucoup d'entreprises ont en effet souhaité continuer à s'adjoindre les services d'un surintendant d'usine, dont les missions sont diverses : recruter les employés, introduire des uniformes, attribuer les postes de travail, s'occuper des installations sanitaires et des vestiaires mis à disposition des salariés, rendre visite aux ouvriers malades, créer des résidences de vacances ou encore organiser les sports et loisirs. Le surintendant travaille souvent en étroite collaboration avec la cantine, pièce maîtresse, tant du point de vue matériel que moral, de la politique sociale qu'il a pour principale mission de coordonner. La remarque d'un inspecteur du travail en 1937 - « la politique sociale ne se résume plus à une cantine de troisième ordre " - souligne l'importance de ces établissements, la présence de cantines devenant synonyme de politique sociale ${ }^{56}$.

51. Chiffres tirés de MM, Annual Report of the Chief Inspector of Factories and Workshops for the Year 1937, 1938, Cmd 5802, p. 93.

52. J. Vernon, Hunger: a Modern History, op. cit., p. 165 sq.

53. J. Melling, British Employers and the Development of Industrial Welfare, c. 1880-1920: an Industrial and Regional Comparison, PhD, Glasgow University, 1980, p. 197-200.

54. MM, Annual Report of the Chief Inspector of Factories and Workshops for the Year 1933, 1934, Cmd 4657, p. 78.

55. MM, Health of Munition Workers Committee Final Report.., op. cit., p. 51-61, et Annual Report of the Chief Inspector of Factories for the Year 1943, Cmd 6563, p. 60.

56. MM, Annual Report of the Chief Inspector of Factories and Workshops for the Year 1937, op. cit., p. 78. 
Avant 1914, l'emploi d'un surintendant d'usine relevait de l'exception, même si, de l'avis des inspectrices, la présence de surintendantes féminines favorisait la propreté, la discipline et la tenue morale de l'entreprise et des employés ${ }^{57}$. Pendant la guerre, quelque 1400 surintendants d'usine ont travaillé sous les ordres du département d'action sociale du ministère des Munitions ${ }^{58}$. Instauré en grande partie pour répondre aux difficultés supposément soulevées par l'afflux d'une main-d'œuvre féminine dans des industries traditionnellement masculines, ce système d'assistance sociale touche également les jeunes garçons et les hommes adultes. Fondée en 1918, la Boys' Welfare Society ${ }^{59}$ est présidée par sir William Beardmore, dont l'entreprise de construction navale avait investi dans des équipements sociaux avant la guerre ; quinze des vingt et un membres de son conseil d'administration sont des chefs d'entreprise. Lorsque la Seconde Guerre mondiale éclate, le nombre de surintendants d'usine n'a que très légèrement progressé, atteignant $1500^{60} .0,6 \%$ des usines employaient un surintendant en 1939 mais il est possible qu'en fait, $2 \%$ de ces établissements aient proposé une cantine, ce qui correspond aux chiffres avancés par un dirigeant d'entreprise en 1924 : d'après lui, 1300 entreprises britanniques offraient ce type de prestations sociales à leurs employés ${ }^{61}$. Néanmoins, la proportion d'ouvriers de l'industrie ayant accès à une cantine est sans doute plus élevée que ces données ne le suggèrent, car il est statistiquement plus probable de trouver des cantines dans les entreprises de grande taille. En 1936, 76,9\% des usines emploient moins de 25 personnes (soit $12,8 \%$ de la force de travail totale). Les cantines se trouvent essentiellement dans les $1,1 \%$ d'usines employant plus de 500 personnes (1 535 usines en 1936). C'est donc plus de $31 \%$ de la main-d'œuvre ouvrière qui avait en fait accès à une cantine sur son lieu de travail ${ }^{62}$.

Le climat économique de l'entre-deux-guerres a indéniablement entravé l'essor des politiques sanitaires et sociales des entreprises. Cela dit, les cantines sont mieux loties que les services médicaux, comme le montrent les dossiers produits par la Confédération des syndicats ${ }^{63}$ qui, entre les deux guerres, dénonce haut et fort l'insuffisance des services médicaux pour les ouvriers et milite énergiquement pour

57. Par exemple, Annual Report of the Chief Inspector of Factories and Workshops for the Year 1911, 1912, Cmd 6293, p. 159. Voir également Annual Report of the Chief Inspector of Factories and Workshops for the Year 1913, op. cit., 1914, p. 101.

58. Le développement de la surintendance d'usine à cette époque est étudié dans A. WoollacotT, "Maternalism, Professionalism and Industrial Welfare Supervisors in WW1 Britain", Women's History Review, Vol. 3, No. 1, 1994, p. 29-56.

59. La société ayant été rebaptisée Industrial Welfare Society en 1919, sa revue changea également de titre et devint en 1920 le Journal of Industrial Welfare.

60. Annual Report of the Chief Inspector of Factories for the Year 1943, op. cit., p. 57 ; Annual Report of the Chief Inspector of Factories and Workshops for the Year 1918, Cmd 340, p. 43.

61. J. Lee, The Principles of Industrial Welfare, Londres, I. Pitman, 1924, p. 2.

62. En 1936, l'Inspection du travail établit des statistiques sur la répartition des usines en fonction de leurs effectifs en s'appuyant sur une enquête menée auprès d'elles; il n'y a pas eu de réponses pour $13 \%$ des usines. Annual Report of the Chief Inspector of Factories and Workshops for the Year 1937, op. cit., p. 122.

63. Créée en 1868, celle-ci débat des questions intéressant les syndicats d'Angleterre et du pays de Galles et, à partir de 1872, vise à influencer la politique gouvernementale à travers sa commission parlementaire. Voir R. M. MarTin, TUC: The Growth of a Pressure Group, 1868-1976, Oxford, Oxford University Press, 1980, et D. F. MacDonald, The State and the Trade Unions, Londres, The Macmillan Press, $2^{e}$ éd., 1976. 
leur extension ${ }^{64}$. Le conseiller médical de la Confédération, sir Thomas Legge, dans une note datée de $1930^{65}$, observe que seule une cinquantaine des 150000 usines alors en fonctionnement en Grande-Bretagne emploient un médecin du travail à plein temps. Les cinq médecins de l'inspection du travail sont incapables de contrôler les conditions de travail dans l'ensemble de ces usines, auxquelles il faut ajouter 130000 ateliers. Ses constatations rappellent aussi que les patrons de l'entre-deux-guerres offrant des cantines à leurs ouvriers se montrent beaucoup moins enclins à leur offrir d'autres services sociaux. Le ralliement du patronat aux cantines d'entreprise tient en partie au fait qu'elles incarnent de manière très efficace une conception de plus en plus répandue de l'hygiénisme industriel promue par la commission sanitaire du ministère des Munitions, mettant en exergue la santé et le bien-être des salariés, tout en minorant les risques sanitaires liés aux procédés industriels et aux produits toxiques. Les cantines jouent un rôle-clé dans ce modèle qui met l'accent sur la diététique, l'exercice physique et l'hygiène - en somme, sur les comportements que les ouvriers peuvent adopter individuellement pour améliorer leur état de santé et ce, avec le généreux soutien de leurs employeurs philanthropes. Cette approche de la santé au travail est le reflet d'un mouvement international plus large, influencé par les difficultés économiques de l'heure, qui insiste sur la promotion de la santé plutôt que sur le traitement de la maladie.

Dans les années 1920, des associations bénévoles telles que le National Council for Mental Hygiene, la People's League of Health, la New Health Society et le Health Education Council concentrent leur discours sur les facteurs environnementaux en soutenant que l'éducation pourrait améliorer l'état sanitaire de la nation ${ }^{66}$. Soulignant les effets salutaires de l'exercice physique, d'une alimentation saine ${ }^{67}$, de l'air frais et du soleil ${ }^{68}$, elles suggèrent que les bâtiments soient conçus en fonction de ces objectifs. Ce message rencontre un écho auprès de l'ancien président de la commission sanitaire, sir George Newman, qui s'efforce de faire améliorer l'environnement dans lequel les ouvriers vivent et travaillent ${ }^{69}$.

Sur le plan des mesures gouvernementales, ces idées se traduisent par la création de dispensaires ${ }^{70}$. Par ailleurs, les propositions d'instauration d'un service médical d'État s'appuient volontiers sur un discours plus préventif que curatif. Dans les

64. Voir V. Long, The Rise and Fall of the Healthy Factory: The Politics of Industrial Health in Britain, 1914-60, Basingstoke, Palgrave Macmillan, 2011, p. 85-129.

65. Centre des archives contemporaines, The University of Warwick, archives du Trades Union Congress (ci-après Archives TUC), manuscrit 292C/140/4, T. LEGGE, "Prevention as a Benefit under the NHI Act", note communiquée à la commission exécutive du TUC, 24 juin 1930.

66. I. Zweiniger-Bargielowska, Managing the Body: Beauty, Health and Fitness in Britain, 18801939, Oxford, Oxford University Press, 2010, p. 151-192 ; ID., "Raising a Nation of 'Good Animals': The New Health Society and Health Education Campaigns in Interwar Britain", Social History of Medicine, Vol. 20, No. 1, 2007, p. 73-89.

67. Voir D. MatLess, "Bodies made of grass made of earth made of bodies: organicism, diet and national health in mid-twentieth-century England", Journal of Historical Geography, Vol. 27, No. 3, 2001, p. 355-376.

68. S. Carter, Rise and Shine: Sunlight, Technology and Health, Oxford, Berg, 2007.

69. S. Sturdy, "Hippocrates and State Medicine...", art. cité.

70. J. Lewis et B. Brookes, "The Peckham Health Centre, PEP, and the Concept of General Practice During the 1930s and 1940s", Medical History, Vol. 27, No. 2, 1983, p. 151-161 ; K. BarLow, "The Peckham Experiment", Medical History, Vol. 29, No. 3, 1985, p. 264-271. 
années 1930, l'Association médicale socialiste se prononce dans plusieurs publications en faveur de la création d'un service public de médecine préventive inspiré des systèmes de santé mis en place dans des pays tels que l'URSS ou la Suède ${ }^{71}$. Ces projets se heurtent néanmoins aux contraintes budgétaires et à la vision obsédante des conséquences néfastes du chômage et de la pauvreté sur la santé physique et mentale ${ }^{72}$. Ces obstacles apparaissent avec évidence à la lecture des travaux de sir George Newman, dont les rapports officiels au ministère de la Santé, tout en relativisant l'importance de la malnutrition et de la morbidité au sein de la population, constatent que les causes premières de la mauvaise santé sont largement économiques et que le ministère n'a pas les moyens d'y remédier ${ }^{73}$.

Importer cette conception englobante de la santé sur le lieu de travail conduit certes à insister sur le rôle de l'alimentation dans la santé des ouvriers, mais aussi à attirer l'attention sur l'importance de l'hygiène personnelle et de l'exercice physique. Les patrons peuvent ainsi affirmer qu'ils sauvegardent la santé de leurs salariés en ouvrant des cantines, en construisant des bâtiments modernes et aérés, tout en laissant entendre qu'il appartient en retour à ces salariés de veiller à leur hygiène de vie. La Société d'éducation à l'hygiène industrielle (Industrial Health Education Society, fondée en Écosse en 1924 sous le nom d'Industrial Educational Council) est l'exemple-type de la manière dont les principes sanitaires de l'entre-deux-guerres, une fois appliqués au monde de l'usine, permettent de mettre sous le boisseau les dangers liés aux procédés industriels et à l'emploi de produits toxiques. Son objectif est d'éduquer les ouvriers à travers des conférences " portant sur les maux et pathologies auxquels ils sont exposés suivant la nature de leur emploi ${ }^{74}$. Néanmoins, nombre des causeries qu'elle organise traitent de thèmes généraux comme la santé dentaire, l'anémie ou la constipation. La Société sollicite le soutien financier des patrons en arguant du fait que la bonne santé des ouvriers augmente le rendement, tout en affirmant que l'ouvrier « est souvent négligent et indifférent aux questions dont sa condition physique dépend au premier chef : son repos, son exercice, son alimentation, pour ne rien dire de l'hygiène générale de son logis ${ }^{75}$.

La Société s'attire d'ailleurs les foudres de sir Thomas Legge qui a assisté à l'une de ses conférences sur le saturnisme. "Rien dans cette conférence ne laissait entendre que les patrons devraient faire quoi que soit, fulmine le conseiller médical de la Confédération des syndicats, pas un mot sur l'utilisation de peintures non toxiques ${ }^{76}$. Nommé inspecteur-médecin principal des fabriques en 1898, il démissionne devant le refus du gouvernement de ratifier la convention de Genève de 1919 interdisant l'usage de peintures au plomb dans l'industrie du bâtiment. "À l'époque où je suis entré à l'Inspection du travail, je me disais : "Allons, tout irait très bien si seulement les ouvriers voulaient bien porter des respirateurs et se

71. Pour une étude détaillée de l'Association médicale socialiste, voir J. STEWART, The Battle for Health: A Political History of the Socialist Medical Association, Aldershot, Ashgate, 1999.

72. C. Webster, "Healthy or Hungry Thirties?", History Workshop Journal, Vol. 13, No. 1, 1982, p. 110-129; H. L. Beales et R. S. Lambert (eds.), Memoirs of the Unemployed, Londres, Victor Gollancz, 1934.

73. C. Webster, "Healthy or Hungry Thirties?”, art. cité.

74. Archives TUC, manuscrit 292/141.1/2, brochure de l'Industrial Education Council, mars 1925.

75. Ibid., manuscrit 292/141.1/2, J. JeRvis, “The Healthy Worker”, tapuscrit.

76. Ibid., manuscrit 292/141/1/3, note de T. Legge, 24 juillet 1930. 
laver les mains" ", raconte Legge en 1930. Lorsqu'il quitte l'inspection du travail, son opinion a considérablement évolué : l'intoxication résulte de l'inhalation de poussières et de vapeurs, elle ne cessera que lorsque celles-ci disparaitront, et " tant que l'employeur n'aura pas tout fait (et "tout", ça veut dire beaucoup), l'ouvrier sera pratiquement impuissant à se protéger $" 77$. Ces convictions, connues sous le nom d'axiomes de Legge, ont été publiées dans un livre posthume, Industrial Maladies ${ }^{78}$. L'Association des fabricants de plomb, qui s'oppose à la ratification de la convention de 1919, avait versé une cotisation de 20 livres sterling à la Société d'éducation à l'hygiène industrielle et lui verse encore une livre supplémentaire pour chaque conférence sur le saturnisme donnée aux ouvriers. Legge en conclut naturellement que l'Association finance la Société pour préserver le statu quo ${ }^{79}$.

Legge est une figure éminente de l'hygiénisme industriel, mais son point de vue est loin d'être unanimement partagé. Ainsi, John Bridge, qui lui succède au poste d'inspecteur-médecin principal des fabriques, défend fréquemment l'idée que le milieu de l'usine est plus sain que les logements des ouvriers. S'il est indéniablement des cas "où l'activité professionnelle engendre des problèmes de santé ", reconnaît-il en 1935, son expérience des conditions de travail en entreprise l'amène à penser que "c'est majoritairement en dehors de l'usine que les problèmes de santé trouvent leur origine $»^{80}$. Les médecins interrogés par la Confédération des syndicats dans le cadre d'une enquête sur la santé des femmes au travail sont également convaincus que l'environnement de l'usine est plus salubre que les habitations des ouvriers et estiment qu'une usine modèle, comportant notamment une cantine, pourrait être un instrument de pédagogie sanitaire. Le docteur Shannon suggère, par exemple, qu'une cantine d'usine pourrait non seulement servir des repas nourrissants, mais également enseigner aux ouvrières « les valeurs nutritionnelles et les différentes façons de donner une alimentation substantielle, qu'elles pourront, une fois ce savoir acquis, fournir à leur famille ». Selon lui, l'anémie est " le plus souvent imputable à des facteurs extérieurs : mauvaise hygiène, nourriture inadaptée et mal choisie, manque d'air frais, de légumes, etc. $»^{81}$.

L'extension des politiques sociales d'entreprise entre les deux guerres n'amène pas pour autant à conclure à une amélioration de la situation sanitaire des ouvriers. L'inspection du travail signale ainsi que, dans les bâtiments industriels les plus anciens, les obligations réglementaires peinent à être remplies et qu'au sein même des établissements modèles, l'instauration d'un service médical va rarement au-delà du minimum exigible par la loi : la présence d'une trousse de secours. Même l'Industrial Welfare Society reconnaît qu'on a parfois procuré du « superflu » en matière de prestations sociales, alors que des problèmes sanitaires fondamentaux n'avaient

77. Ibid., manuscrit 292/140/1, "Industrial Disease with special reference to Silicosis" : discours prononcé par T. Legge devant le Conseil national des industries céramiques, 24 mars 1930.

78. T. Legge, Industrial Maladies, Oxford, Oxford University Press, 1934.

79. Archives TUC, manuscrit 292/141.1/3, note de T. Legge portant sur la réunion du 22 juillet 1930 du comité exécutif de la Société pour l'éducation à l'hygiène industrielle, envoyée le 24 juillet 1930. Pour une étude de cette société et de ses rapports avec le TUC, voir V. Long, The Rise and Fall..., op. cit., p. 113-123.

80. MM, Annual Report of the Chief Inspector of Factories and Workshops for the Year 1934, 1935, Cmd 4931, p. 51.

81. Archives TUC, manuscrit 292/134.1/5, rapport tapuscrit, 1938. 
pas été traités ${ }^{82}$. Industrial Welfare, la revue de l'association, présente d'ailleurs de plus en plus les objectifs de l'action sociale patronale sous l'angle du taylorisme, du rendement et de la gestion de la main-d'œuvre. La thèse de Joseph Melling et Helen Jones selon laquelle la politique sociale devient un nouveau moyen de renforcer le contrôle de la direction sur les ouvriers prend dès lors toute sa force ${ }^{83}$. Melling rappelle la façon dont le patronat s'en est servi pour saper le syndicalisme entre les années 1880 et les années 1920 en proposant des systèmes d'épargne alternatifs visant en priorité les catégories d'ouvriers qui occupaient des postes-clés dans la division du travail. Après avoir constaté l'influence de l'interventionnisme gouvernemental sur les stratégies d'action sociale du patronat durant la Première Guerre mondiale, Melling affirme qu'un grand nombre de patrons en sont venus "à considérer l'implantation d'une "cantine efficace et bien gérée" comme une incitation au rendement et à un "plus haut degré de satisfaction" chez les ouvriers ". Les patrons disposent également de moyens simples, tels que l'expulsion des logements ouvriers, pour museler les comportements qu'ils jugent antisociaux et improductifs ${ }^{84}$. « La dimension disciplinaire et autoritaire " demeure donc prégnante au sein de la cantine d'entreprise ${ }^{85}$. Les ouvriers de certains secteurs n'accueillent d'ailleurs pas sans réserve la création de cantines sur leur lieu de travail. Nick Hayes, par exemple, signale que les ouvriers du bâtiment refusent les cantines et salles de repas, où ils voient une atteinte à leur virilité ${ }^{86}$.

Les équipements sociaux comme les cantines sont également utiles aux patrons qui cherchent à repousser l'adoption d'une nouvelle législation sur la santé et la sécurité dans les entreprises en se faisant les chantres du volontarisme. Comme l'a montré Helen Jones, cela a pour conséquence de creuser l'écart entre les entreprises modèles et celles qui se conforment à peine aux exigences de la loi sur les manufactures de $1901^{87}$ qui, jusqu'à la nouvelle loi de 1937, encadre les conditions de travail dans les usines et ateliers. Cette loi stipule que la ventilation doit être "suffisante", la température " raisonnable ", les bâtiments maintenus en état de " propreté "; des " moyens adéquats » doivent également être employés pour drainer les sols, tandis que les installations sanitaires doivent être "suffisantes et adaptées ". L'imprécision des termes n'est pas sans poser problème aux inspecteurs. Une inspectrice se souvient dans ses mémoires : "Il était souvent difficile de convaincre les magistrats d'un tribunal qu'en poursuivant un employeur obstiné nous n'étions pas simplement "tatillonnes" ou assoiffées d'une orgie de grand nettoyage à ses frais ${ }^{88}$.

82. C. U. Kerr, "Some Health Aspects of a Welfare Scheme", Industrial Welfare, Vol. 5, September 1923, p. 264-266.

83. J. Melling, "Employers, Industrial Welfare, and the Struggle for Work-Place Control in British Industry, 1880-1920", in H. F. Gospel et C. R. Littler (eds.), Management and Labour in British Business Strategies, Londres, Heinemann, 1983, p. 55-81, et J. Melling, British employers..., thèse citée.

84. Voir, par exemple, l'étude de Brian Lewis sur les pratiques en vigueur à Port Sunlight, le village appartenant à l'entreprise de lord Leverhulme : B. Lewis, So Clean: Lord Leverhulme, Soap and Civilization, Manchester, Manchester University Press, 2008, p. 120-129.

85. J. Melling, British Employers..., thèse citée, p. 159.

86. N. HAYES, "Did Manual Workers Want Industrial Welfare? Canteens, Latrines and Masculinity on British Building Sites, 1918-1970", Journal of Social History, Vol. 35, 2002, p. 637-658.

87. H. Jones, "Employers' Welfare Schemes and Industrial Relations in Inter-War Britain", Business History, Vol. 25, No. 1, 1983, p. 61-75.

88. R. SQuire, Thirty Years in the Public Service: An Industrial Retrospect, Londres, Nisbet \& Co, 1927, p. 45. 
En 1937, l'inspection du travail affirme que « jamais dans l'histoire des lois industrielles les relations entre [elle] et les deux principaux partenaires du progrès industriel (le patronat et les ouvriers) n'auront été aussi étroites et chaleureuses ${ }^{89}$. Derrière cette rhétorique de la coopération se cache la difficulté, au même moment, d'imposer des progrès par la voie législative. Beaucoup d'organisations patronales résistent vigoureusement à l'instauration de normes minimales en arguant de la situation économique désastreuse et des coûts que ces normes engendreraient. La tendance à l'adoption de normes volontaires, auxquelles tous les patrons ne se plient pas, provoque de fortes disparités entre établissements ${ }^{90}$. Dressant l'inventaire des mesures sociales spontanément mises en ouvre par certaines entreprises, la Confédération des syndicats reconnaît en 1932 que beaucoup reste à faire dans les petits établissements où les conditions de travail demeurent déplorables. Néanmoins, elle est convaincue que le développement de prestations sociales de plus en plus élaborées au sein d'entreprises isolées est "de nature à entraver le progrès vers ce service de santé national, unifié, complet et adéquat, qui doit être regardé comme un idéal $»^{91}$.

La loi de 1937, qui encadre des dispositions jusqu'alors laissées au bon vouloir des employeurs, n'est votée qu'après plus d'une décennie de lutte, les propositions de loi présentées par le parti travailliste en 1924 et 1926 ayant été rejetées tandis que les organisations patronales cherchaient à " obtenir l'abandon, le report ou l'adoucissement de la loi sur les manufactures ${ }^{92}$. On s'explique mieux que les cantines d'entreprise se soient multipliées en dépit (ou peut-être à cause) des efforts du patronat pour occulter les risques sanitaires liés aux procédés de fabrication et pour retarder toute intervention législative. La décision d'un employeur de créer une cantine ne vaut ainsi pas engagement de protéger ses salariés des maladies professionnelles, ce qu'illustre un article très révélateur de la revue Industrial Welfare publié en avril 1928 : comme à l'accoutumée, il offre une vitrine élogieuse aux cantines, vestiaires et équipements sportifs modèles du fabricant d'amiante Turner Brothers Asbestos Company ${ }^{93}$. Ce n'est évidemment pas une coïncidence si le terme d'asbestose a été forgé à peine un an plus tôt pour désigner la maladie qui a emporté un des salariés de cette entreprise, incitant le gouvernement à commander une étude sur la santé des ouvriers dans le secteur de l'amiante textile ${ }^{94}$.

\section{Cantines et rationalisation industrielle}

Les débats sur la fonction remplie par les cantines d'entreprise mettent donc en lumière deux visions contradictoires de l'hygiénisme industriel pendant l'entre-deuxguerres : un modèle englobant, qui insiste sur la responsabilisation du travailleur,

89. MM, Annual Report of the Chief Inspector of Factories and Workshops for the Year 1937, 1938, Cmd 5802, p. 11.

90. MM, Annual Report of the Chief Inspector of Factories and Workshops for the Year 1930, 1931, Cmd 3297, p. 11.

91. Archives TUC, manuscrit 292C/146.9/6, comité exécutif du TUC, "The Effect of Welfare Work on Trade Unionism", tapuscrit, 24 mai 1932.

92. Ibid., manuscrit 292C/140/1, département de recherche du TUC, T. LegGe, "Factories Bill", tapuscrit daté du $1^{\text {er février } 1927 .}$

93. C. U. KerR, "Welfare Work in a Lancashire Asbestos Factory", Industrial Welfare, Vol. 10, April 1928, p. $109-114$

94. G. Tweedale, Magic Mineral to Killer Dust: Turner \& Newall and the Asbestos Hazard, Oxford, Oxford University Press, 2000, p. 11-22. 
et un modèle concurrent, défendu par la Confédération des syndicats, centré sur la responsabilisation du patron, tenu de protéger ses salariés des risques inhérents aux procédés de fabrication et aux matériaux employés. Les contradictions que l'on peut relever dans les débats sur l'aménagement et la fonction des cantines témoignent également d'un infléchissement plus global du discours sur les relations sociales pendant cette période. Au lendemain de la Première Guerre mondiale, les surintendants font d'abord l'apologie des cantines en les présentant comme un moyen d'endiguer la marée montante de la " guerre sociale " qui a caractérisé la décennie précédente $^{95}$. Le problème, expliquent-ils, tient à l'essor rapide et désordonné du système de production en usine et à la nature de plus en plus limitée du rôle dévolu à chaque ouvrier qui n'est plus qu'un rouage anonyme dans une machine, d'où une inquiétude bien résumée dans l'épigraphe d'un volume édité par l'Institute of Welfare Workers en 1925: "Dans un système de production à grande échelle, l'individu sera souvent le grand oublié ${ }^{96}$. La disparition manifeste des bonnes relations qui, selon les surintendants, unissaient autrefois la direction et le personnel des petites usines laisse en présence les deux partis, qui cherchent donc à résoudre leurs différends en recourant à l'action collective.

Même si les surintendants dressent un tableau idéalisé de l'harmonie qui pouvait régner autrefois dans les entreprises, il n'est pas douteux que la déqualification et l'intensification du travail aient accentué les tensions sociales ${ }^{97}$. Pour eux, les cantines devraient offrir un répit permettant d'échapper à la rationalisation industrielle. Nombre d'entre eux rejettent les considérations utilitaires qui avaient prévalu pendant la guerre. Au contraire, les auteurs jugent souhaitable d'aménager des cantines où l'on se sente comme chez soi ; une conception soignée de l'espace industriel pourra influer sur l'état d'esprit et le comportement des salariés ${ }^{98}$. Un " grand hangar sans charme et sans confort faisant office de salle à manger " n'encouragera pas les ouvriers à se conduire comme s'ils étaient chez eux, met en garde Dorothea Proud dans son livre de 1916 sur l'action sociale en entreprise ${ }^{99}$. Elle recommande d'équiper les cantines de sièges confortables, de nappes, de fleurs et d'images, dans le but de créer un espace agréable "qui n'évoque en rien un lieu de labeur " ${ }^{100}$. Dans les années 1920, Industrial Welfare incite les employeurs à construire des cantines qui ressembleraient à une salle à manger familiale et publie des photographies de nouvelles cantines dans lesquelles un effort a été fait pour modeler les comportements et créer des espaces de restauration dûment sexués. En 1923, par exemple, la revue publie un reportage sur la nouvelle cantine de la centrale électrique de

95. The Times employa pour la première fois l'expression de "guerre industrielle " (industrial war) en 1876 : il estimait que, dans la mesure où les syndicats s'étaient confédérés, il était temps pour le patronat d'en faire autant "en vue de sa propre sauvegarde ": "Employers and Trade Unions", The Times, 26 février 1876, p. 5. L'emploi de cette expression dans le journal culmina dans les années 1910.

96. E. T. KelLy (ed.), Welfare Work in Industry, by members of the Institute of Welfare Workers, Londres, Pitman, 1925, p. 1.

97. Voir A. McIvor, A History of Work in Britain, 1880-1950, Basingstoke, Palgrave, 2001, p. 43-78.

98. Cette question est développée dans V. LonG, "Industrial homes, domestic factories : the convergence of public and private space in interwar Britain", Journal of British Studies, Vol. 50, No. 2, April 2011, p. 434-464.

99. E. D. Proud, Welfare Work: Employers' Experiments for Improving Working Conditions in Factories, Londres, G. Bells \& Sons, 1916, p. 122.

100. Ibid, p. 125. 
Dalmarnock. On y trouve des boiseries et des gravures au mur. "L'ensemble crée une atmosphère qui rappelle l'intimité du foyer", proclame-t-elle ${ }^{101}$.

De plus en plus, les espaces dédiés à la restauration sont intégrés à des bâtiments qui accueillent d'autres équipements sociaux. Les réfectoires du Wallace Scott Tailoring Institute, près de Glasgow, font forte impression à C. U. Kerr, contributrice régulière d'Industrial Welfare, qui vante « l'atmosphère d'harmonie et de bien-être " qui y règne ${ }^{102}$. Cette cantine possède un parquet en érable et une estrade, de sorte qu'on peut y donner des bals, des pièces de théâtre, des concerts et y projeter des films. Des salles de repos et de lecture destinées au personnel féminin sont contiguës à la cantine, de même qu'un fumoir pour les hommes. "Les repas pris dans un tel cadre doivent être aussi réparateurs pour le corps que pour l'esprit " ${ }^{103}$, commente C. U. Kerr, qui reprend pratiquement mot pour mot cette phrase pour décrire les réfectoires de la Shredded Wheat Factory à Welwyn Garden City ${ }^{104}$. Selon la journaliste, cette cantine, ouverte en 1926, pourrait favoriser l'harmonie sociale en intégrant chaque employé à la "grande famille Shredded Wheat". "Chaque individu est traité comme un associé dans l'entreprise ", souligne-t-elle, et ce principe est illustré par le réfectoire, où tous les membres du personnel se voient servir des repas gratuits - « un bon investissement tant pour la santé que pour la productivité ${ }^{105}$.

Cependant, bien que l'on cherche à faire des cantines des espaces sociaux unificateurs, en pratique leur agencement et leur fonctionnement séparent fréquemment les salariés en fonction de leur sexe et de leur classe sociale. À Port Sunlight, les divisions hiérarchiques s'inscrivent dans les diverses cantines de l'entreprise après la mort de lord Leverhulme en 1925. Ouvriers, employés, administrateurs, cadres supérieurs et direction disposent chacun de leur cantine et les promotions s'accompagnent d'une ascension dans la hiérarchie des cantines ${ }^{106}$. De même, le bâtiment construit par Turner Brothers à Trafford Park est conçu pour trier les salariés en fonction de leur statut. Il comprend un réfectoire principal, qu'Industrial Welfare qualifie de " majestueux ", quoique la photographie d'illustration évoque davantage un hangar impersonnel dans lequel les piliers métalliques sont clairement apparents et où de longues tables sur tréteaux pour huit personnes s'alignent en rangées serrées et régulières. On y trouve du lambris sur le bas des murs et de la peinture crème au-dessus, mais on ne voit ni nappes, ni fleurs, ni plantes ou autre élément de décoration. Comme beaucoup d'autres à l'époque, cette cantine possède un parquet en bois massif, ce qui permet de l'utiliser pour danser, bien que la poussière d'amiante tassée sous les lames pour insonoriser la salle rende une telle activité dangereuse $\mathrm{e}^{107}$. Les employés de bureau disposent de deux autres réfectoires, un pour les femmes, un pour les hommes, en haut de deux escaliers distincts. Enfin, on trouve à l'étage

101. R. B. M., "An 'All-Electric' Canteen”, Journal of Industrial Welfare, Vol. 5, May 1923, p. 124-128, p. 124.

102. C.U. KerR, "A Model Factory in Scotland", Industrial Welfare, Vol. 6, January 1924, p. 16-21, p. 18.

103. Ibid.

104. C. U. KerR, "A New Model Factory”, Industrial Welfare, Vol. 8, May 1926, p. 160-164, p. 162.

105. Ibid.

106. B. Lewis, So Clean..., op. cit., p. 122.

107. C. U. KerR, "Welfare Work in a Lancashire Asbestos Factory", art. cité, p. 110-111. 
un espace théoriquement accessible à tout le personnel, mais que l'article désigne comme une "salle à manger privée ", avec des nappes et une décoration en plaques de ciment-amiante "peintes dans des nuances pastels et soulignées de moulures en bois émaillé ${ }^{108}$. Ce restaurant facture 1 shilling et 6 pence par tête quand le restaurant ordinaire coûte 8 pence. L'article explique qu'une telle organisation offre à tous les employés « le privilège de côtoyer les plus hauts dirigeants de l'entreprise ", tout en faisant l'économie « des aigreurs que l'on peut parfois ressentir devant de petites différences de traitement ou lorsque des inégalités de prestige et de statut, réelles ou imaginaires, entrent en ligne de compte $»^{109}$.

Les cantines sont également aménagées de manière à canaliser le flux des employés depuis leur entrée jusqu'à leur départ, en passant par le choix et le paiement des plats, puis l'installation à table. Ainsi, la cantine de la London General Omnibus Company, ouverte en 1927, est conçue pour faciliter « la fourniture de centaines de repas chauds en quelques minutes $»^{110}$. On y trouve un long comptoir avec un écran de métal poli derrière lequel des armoires à vapeur mesurant jusqu'à quinze mètres de long conservent un assortiment de plats chauds. Cet espace alterne avec des parties non chauffées où l'on trouve des plats froids parmi lesquels les employés font leur choix avant d'aller s'attabler dans le réfectoire. De toute évidence, on n'a pas cherché à créer une oasis de confort et de détente permettant de s'abstraire de la pression de la rationalisation industrielle ; au contraire, les principes de la rationalisation ont été appliqués au sein même de la cantine. Il s'agit là d'une illustration du type d'usine modèle moderniste qui s'appuie sur des styles architecturaux et des matériaux de construction nouveaux pour mieux rejeter le modèle de l'usine paternaliste et familiale ${ }^{111}$.

L'implantation de cantines au sein d'un grand nombre d'usines britanniques pendant la Première Guerre mondiale a été le fruit de l'intervention du gouvernement, qui poursuivait d'abord des objectifs militaires. L'alimentation des ouvriers redevint une préoccupation gouvernementale majeure pendant la Seconde Guerre mondiale et la demande de cantines dépassa celle qu'on avait pu constater pendant le conflit précédent, car le rationnement, les dommages subis par les logements des ouvriers lors des bombardements, l'accroissement du taux d'emploi des femmes mariées, le travail posté et la réduction des temps de pause-repas rendaient plus difficile l'alimentation des ouvriers. En 1940, 64 \% des fabriques travaillant essentiellement à fournir des munitions et employant plus de 500 personnes ont ouvert des cantines, et dans certaines régions du sud de l'Angleterre, cette proportion peut atteindre $90 \%{ }^{112}$. À la suite du décret-loi de 1940 sur les cantines d'entreprise, des conseillers ont été désignés pour aider les employeurs à surmonter les défis posés par l'agencement, l'équipement, l'embauche de personnel, l'établissement de menus, la préparation des repas et les enjeux nutritionnels liés à la restauration collective. Le nombre de cantines passa ainsi de 5695 en décembre 1941 à 11630 en décembre $1944^{113}$.

108. Ibid., p. 112.

109. Loc. cit.

110. F. C., "Equipment Notes: Works Canteens", Industrial Welfare, Vol. 9, 1927, p. 345-348, p. 346.

111. Sur les nouveaux matériaux employés dans la construction d'usines, voir M. STRATTON et B. Trinder, Twentieth Century Industrial Archaeology, Londres, Taylor \& Francis, 2000, p. 7-14. Sur l'idéal d'une usine " où l'on se sente comme à la maison », voir V. LoNG, "Industrial homes, domestic factories...", art. cité.

112. MM, Annual Report of the Chief Inspector of Factories for the Year 1940, 1941, Cmd 6316, p. 14.

113. MM, Annual Report of the Chief Inspector of Factories for the Year 1944, 1945, Cmd 6698, p. 84. 
Il serait pourtant trompeur d'affirmer que l'existence de cantines d'entreprise en Grande-Bretagne ne fut que le résultat de l'intervention étatique pendant les deux guerres. Le patronat n'a pas abandonné les cantines à la fin des hostilités mais, alors que, en temps de guerre, la préoccupation principale des autorités était de soutenir la productivité des ouvriers en leur procurant une alimentation nourrissante, le patronat de l'entre-deux-guerres espérait que les cantines seraient un moyen de désamorcer les tensions sociales grandissantes. Les surintendants d'usine considéraient également la cantine comme un élément-clé dans un programme d'action sociale centré sur l'individu et susceptible de combattre le sentiment d'aliénation provoqué, pensait-on, par la fragmentation des processus de production et la hausse des effectifs. Présentée comme un refuge domestique isolé du lieu de production, la cantine égalitaire dans laquelle la « famille » des employés aurait communié en prenant ses repas dans un même espace se révéla rapidement une chimère. D’une part, l'organisation des cantines reflétait les hiérarchies en vigueur au sein des entreprises : souvent, différentes salles étaient destinées aux différents échelons et le rang que chacun occupait était signifié par la présence ou l'absence de nappes, d'œuvres d'art, de tables de taille variable et de serveurs. Par ailleurs, une cantine à l'atmosphère intime était considérée comme un instrument disciplinaire pouvant servir à réfréner les comportements perturbateurs et à inculquer des habitudes d'ordre.

Durant l'entre-deux-guerres, l'idéal de la salle à manger familiale fut progressivement abandonné par les architectes modernistes qui, influencés par les idéaux de la gestion scientifique, ont cherché à rationaliser l'espace de la cantine avec toujours les mêmes objectifs : augmenter le rendement et contenir l'agitation ouvrière en modelant les comportements individuels et la vie sociale dans l'enceinte de l'entreprise $^{114}$. La recherche du rendement séduisait une direction soucieuse de profits bien plus que la conception englobante de la santé et du bien-être au travail défendue par les surintendants. On peut donc se demander si, loin d'offrir un dépassement du processus de rationalisation industrielle et la dépersonnalisation de l'ouvrier qui en découlait, les cantines n'étaient pas l'expression même de la rationalisation industrielle puisqu'elles servaient à lutter contre l'indiscipline tout en assurant l'entretien du facteur humain de la production.

L'idéal de santé au travail qui a pris corps durant la Première Guerre mondiale et dont les cantines d'entreprise sont le reflet permet d'expliquer pourquoi celles-ci sont devenues la pierre angulaire de l'action sociale patronale pendant l'entre-deuxguerres. Les cantines matérialisaient en effet une vision englobante de la santé au travail, où l'accent était mis sur la responsabilité de l'ouvrier dans l'entretien de sa santé à travers l'alimentation, l'hygiène et l'exercice physique. Minorant la responsabilité de l'employeur dans la santé de ses employés et occultant les risques sanitaires propres au lieu de production industriel, elles ont constitué un outil efficace aux mains de ceux qui voulaient repousser l'adoption d'une législation sur la santé et la sécurité dans les entreprises. Une telle observation suggère à quel point l'étude des prestations sanitaires et sociales est complémentaire de celles traitant des risques auxquels les produits toxiques et les procédés dangereux exposent les salariés.

114. M. F. Guillen, The Taylorized Beauty of the Mechanical: Scientific Management and the Rise of Modernist Architecture, Princeton, Princeton University Press, 2006, p. 33-44. 